\title{
Evaluation of a multiplex polymerase chain reaction for early diagnosis of ventriculostomy-related infections
}

\author{
Claire L. Gordon, MBBS, $, 1,6$ Rafal Tokarz, PhD, ${ }^{2}$ Thomas Briese, PhD, ${ }^{2,3}$ W. lan Lipkin, MD, ${ }^{2}$ \\ Komal Jain, ${ }^{2}$ Susan Whittier, PhD, ${ }^{5}$ Jayesh Shah, ${ }^{1}$ E. Sander Connolly, MD, ${ }^{4}$ \\ and Michael T. Yin, MD'
}

\begin{abstract}
1'Division of Infectious Disease, Department of Medicine, and ${ }^{4}$ Department of Neurosurgery, Columbia University Medical Center; ${ }^{2}$ Center for Infection and Immunity and ${ }^{3}$ Department of Epidemiology, Mailman School of Public Health, Columbia University Medical Center; ${ }^{5}$ Clinical Microbiology Service, NewYork-Presbyterian Hospital, New York, New York; and ${ }^{6}$ Department of Medicine, University of Melbourne, Victoria, Australia
\end{abstract}

OBJECT Diagnosis of ventriculostomy-related infections (VRIs) is challenging due to the lack of rapid, sensitive assays for pathogen detection. The authors report the development of a multiplex polymerase chain reaction (PCR) assay for differential diagnosis of common VRI pathogens.

METHODS MassTag PCR was used to develop a multiplex assay for detection of 11 VRI pathogens. The assay was established and optimized using cloned template standards and spiked samples and was then evaluated on CSF specimens from ventricular drains. Subjects were grouped into definite VRI, possible VRI, or no VRI based on conventional microbiology, CSF evaluation, and clinical parameters.

RESULTS CSF specimens were obtained from 45 subjects (median age 49 years, interquartile range 32-63 years; 51\% were male). The assay detected 10-100 genome copies. It detected a pathogen in 100\% (6 of 6 ) of definite VRI cases in which a pathogen targeted by the assay was present; these represented $67 \%$ of all definite VRIs ( 6 of 9 ). Among subjects with a possible VRI, the assay detected a pathogen in $29 \%$ (5 of 17). In subjects without overt infection the presence of a pathogen was detected in $32 \%$ of subjects (6 of 19), albeit with lower signal compared with the VRI group.

CONCLUSIONS MassTag PCR enabled parallel testing of CSF specimens for 11 pathogens of VRI. The high sensitivity of PCR combined with possible device colonization, specimen contamination, and concurrent antibiotic treatments limit the clinical value of the assay, similar to other current diagnostic approaches. With further optimization, multiplex PCR may provide timely identification of multiple possible VRI pathogens and guide management, complementing classic culture approaches.

http://thejns.org/doi/abs/10.3171/2014.11.JNS141036

KEY WORDS ventriculostomy infection; PCR; diagnosis

$\mathrm{V}$ ENTRICULOSTOMY devices are used in the management of acute hydrocephalus by providing therapeutic drainage of CSF and intracranial pressure monitoring. However, ventriculostomy-related infections (VRIs) frequently occur, causing significant morbidity and mortality. ${ }^{6}$ Diagnosis of ventriculomeningitis is difficult because similar clinical and CSF parameters are often present after intraventricular hemorrhage and neurosur- gery. ${ }^{5}$ To reduce the risk of VRIs, many institutions use prolonged antibiotic "prophylaxis" during the use of ventriculostomy devices despite limited evidence of efficacy. ${ }^{7}$ Definitive diagnostic culture results can take several days and are often negative because of antibiotic inhibition. ${ }^{8,10}$ Given the inability to obtain a rapid, sensitive diagnosis and the potential for serious neurological sequelae associated with delayed treatment, most clinicians treat em-

\footnotetext{
ABBREVIATIONS CoNS = coagulase-negative Staphylococcus spp.; MRSA = methicillin-resistant Staphylococcus aureus; MSSA = methicillin-sensitive S. aureus; NYP = NewYork-Presbyterian; PCR = polymerase chain reaction; VRI = ventriculostomy-related infection.

SUBMITTED May 9, 2014. ACCEPTED November 6, 2014.

INCLUDE WHEN CITING Published online May 29, 2015; DOI: 10.3171/2014.11.JNS141036.

DISCLOSURE This work was supported by the National Center for Advancing Translational Sciences, National Institutes of Health, through Grant Number UL1 TR000040, formerly the National Center for Research Resources, Grant Number UL1 RR024156, National Institutes of Health award AI057158 and the US Department of Defense. The authors report no conflict of interest concerning the materials or methods used in this study or the findings specified in this paper.
} 
pirically with broad-spectrum antibiotics when there is clinical suspicion of a VRI.$^{14}$ However, these strategies promote the selection of resistant microorganisms ${ }^{7}$ and expose the patient to the risks of potentially unnecessary antibiotics. Polymerase chain reaction (PCR) assays allow for rapid and specific amplification of microbial nucleic acids. We have developed a multiplex PCR primer panel for the early differential diagnosis of VRIs using bacterial and fungal primers. This panel was used to build a MassTag PCR assay wherein microbial gene targets are recognized by the presence of tags with different molecular weights attached to agent-specific primers. ${ }^{2}$

\section{Methods}

\section{Development of Multiplex PCR Assay for VRIs}

A review of culture-positive CSF specimens obtained from ventriculostomies performed at NewYork-Presbyterian Hospital (NYP)/Columbia University Medical Center between 2001 and 2006 was performed to determine common VRI pathogens. Bacteriological cultures of CSF specimens were performed in accordance with standard laboratory protocol. Specimens were inoculated onto 3 agar plates (colistin-nalidixic acid with 5\% sheep blood, chocolate, and MacConkey, BBL) and an aliquot was added to prereduced thioglycollate broth (BBL). Media were incubated at $35^{\circ} \mathrm{C}$ in an atmosphere of $5 \% \mathrm{CO}_{2}$. Plates were examined by a technologist daily for up to 4 days, and broths were examined daily for 14 days and subcultured when cloudy. All bacterial growth was identified to species level using VITEK TWO (bioMérieux) for gramnegative bacteria and MicroScan Walk-Away (Dade Behring Inc.) for gram-positive cocci.

An 11-plex MassTag primer panel targeting the most common VRI pathogens was assembled (Table 1). PCR primers were designed as previously described ${ }^{2}$ and optimized using targeted sequences cloned into pCR2.1-TOPO (Invitrogen) or samples with $10^{8}$ live organisms per milliliter of lysis buffer (NucliSENS, bioMérieux) obtained from the Clinical Microbiology Service. Samples were subjected to PCR amplification with a multiplex PCR kit (Qiagen), primers at $0.5 \mu \mathrm{M}$ each in a final reaction volume of $20 \mu \mathrm{l}$, and the following cycling protocol: an annealing step with a temperature reduction in $1^{\circ} \mathrm{C}$ increments from $65^{\circ} \mathrm{C}$ to $58^{\circ} \mathrm{C}$ during the first 8 cycles and then continuing with a cycling profile of $94^{\circ} \mathrm{C}$ for 30 seconds, $58^{\circ} \mathrm{C}$ for 30 seconds, and $72^{\circ} \mathrm{C}$ for 30 seconds for 34 cycles in an MJ PTC200 thermal cycler (MJ Research). Results from the MassTag PCR were obtained within 9 hours (range 8-9 hours), requiring approximately 180 minutes (range 150-210 minutes) of hands-on time when processing a 96well plate with up to 69 samples from extraction to result (the remaining wells are blanks and quantitation standards for each target). The limit of detection for the assay was 10-100 genome copies per reaction for all pathogens, as determined by serial dilution of nucleic acid extracted from the samples containing $10^{8}$ live organisms per milliliter. Assay limitations have been described previously. ${ }^{2}$ Definitive identification of pathogen(s) detected by the multiplex PCR assay was achieved through subsequent specific single-plex PCR amplification and sequencing of the product.

\section{Assessment of Assay Performance Using Clinical Specimens}

The primer panel was evaluated in 45 CSF specimens collected from ventricular drains sent to the NYP Clinical Microbiology Service for culture diagnosis of VRIs from January 2008 to June 2010. A technician in the Clinical Microbiology Service would aliquot $250 \mu \mathrm{l}$ of CSF into $750 \mu \mathrm{l}$ of lysis buffer (NucliSENS, bioMérieux) under sterile conditions for this study, if sufficient CSF was available. All specimens were stored at $-70^{\circ} \mathrm{C}$ and batched for PCR assay testing. This study included all specimens that were collected from ventricular drains and saved by the Clinical Microbiology Service during this period.

Retrospective laboratory and chart review was performed to determine CSF and clinical parameters, and to categorize subjects into the following clinical diagnoses based on accepted definitions: $\left.{ }^{6} 1\right)$ definite VRI, 2) possible VRI, and 3) no VRI. Definite VRI was defined by a progressively declining CSF glucose level, increasing CSF protein profiles, advancing CSF pleocytosis, one or more positive CSF cultures or Gram stains, and clinical features such as fever and signs of meningitis, including nuchal rigidity, photophobia, decreased mental status, seizures, or moribund appearance. Possible VRI was defined by a progressively declining CSF glucose level, increasing CSF protein profiles, and advancing CSF pleocytosis with clinical signs and symptoms, in the absence of positive CSF cultures or Gram stain. No VRI was defined by expected CSF glucose, protein, or cell count; lack of clinical signs and symptoms of infection; and lack of a positive culture or Gram stain. Absolute criteria for acceptable CSF glucose and protein levels or cell counts were not specified because these parameters vary in a predictable manner, depending on the given clinical situation. ${ }^{6}$ In 3 instances, 2 successive samples from the same subject were available; only the first sample was included in the main analysis. During the period of study, both conventional ventricular drains and ventricular drains coated with rifampin and minocycline were used; however, records for catheters used in individual subjects were not available. Practice in our institution often included prolonged antibiotic prophylaxis with intravenous cefazolin while the drain was in situ, ${ }^{15}$ and data on antibiotic use less than 24 hours prior to the collection of the CSF specimen were collected. Data on indication for, or duration of, ventricular drain placement were not collected on these subjects. However, from a prior study from our institution, the primary indications for ventricular drain placement were subarachnoid hemorrhage $(46 \%)$, intracranial hypertension $(30 \%)$, tumor $(21 \%)$, and trauma (3\%), and the average duration of drain placement was $8.2 \pm 6.9$ days. $^{15}$

\section{Results}

The median age of the 45 subjects with CSF specimens analyzed in this study was 49 years; (interquartile range 32-63 years); 51\% (23 of 45) were male (Table 2). Presence of signs and symptoms of VRI, antibiotic administration less than 24 hours prior to CSF sample collection, and relevant CSF parameters for VRI for each subject are shown in Table 2. Among subjects with a definite VRI, the 
TABLE 1. VRI pathogens and gene targets

\begin{tabular}{llll}
\hline \multicolumn{1}{c}{ Organism } & Target Gene & \multicolumn{1}{c}{ Forward Primer } & Reverse Primer \\
\hline Staphylococcus (genus level) & $16 \mathrm{~S} r$ RNA & CCATCATTAAGTTGGGCACTCTAAGTT & GGTTTCGCTGCCCTTTGTATTGT \\
\hline Staphylococcus aureus & $\mathrm{femA}$ & TTCACGCAAACTGTTGGCCACTA & GGTAACAGGTACAGCAGTAAGTAAGCAA \\
\hline MRSA & $\mathrm{mecA}$ & CCTTGTCCGTAACCTGAATCAGCT & GCTAGAGTAGCACTCGAATTAGGCAGT \\
\hline Propionibacterium acnes & $\mathrm{recA}$ & GCAGCAGATCGAGAAGCAGCA & GATCTCCACGATTCTGCCACGT \\
\hline Enterococcus sp. & $16 \mathrm{~S} r$ RNA & GGGGATAACACTTGGAAACAGGT & TCCATCCATCAGCGACACCCGAA \\
\hline Pseudomonas aeruginosa & ExoS & GTCAACTGGTGCTCGACGCAA & CGATACTCTGCTGACCTCGCTCTCT \\
\hline Enterobacter sp. & ompA & GCGCWGACTCCAGCAACARCAT & RCCGTCGCCGATGTTTAA \\
\hline Acinetobacter baumannii & OXA-51 & GCTTCCGCTATTCCRGTTTATCA & CGACTTGGGTACCGATATCTGCATT \\
\hline Serratia marcescens & ompA & GGCCGTACTGGTCAGCGTCT & GCCCAGGCTCAGCATGGT \\
\hline Klebsiella sp. & ompA & GCTTCTIGGITTTCCCGTA & ACGGGTTGCGATGTCACGAGTAACA \\
\hline Candida albicans & CaAGM1 & ACCAGTAGGAGTACAACGAACAGGAA & ATTTCATTGAATATTGGTGGGTTCA \\
\hline
\end{tabular}

MassTag PCR assay detected a microbial sequence in 6 of 6 cases $(100 \%)$ for which a pathogen targeted by the assay was present, and 6 of 9 cases (67\%) overall (Table 3). In 3 cases, Morganella morganii, Streptococcus parasanguinis, and Micrococcus sp. were detected by culture but not by the MassTag PCR, since the assay did not include specific primers for these organisms. Among subjects with a possible VRI, we detected at least one potential pathogen in 5 of 17 cases (29\%) (Table 3). In subjects with no VRI, a nucleic acid of an organism was detected in 6 of 19 cases $(32 \%)$.

For methicillin-sensitive Staphylococcus aureus (MSSA), one of the most commonly detected pathogens, the positive samples with highest signal relative to the quantitative positive controls (and thus those with the highest pathogen load) all occurred in the definite VRI group; considerably lower signal relative to the quantitative positive controls was evident in the positive samples from the group without VRI (Table 2). Similarly, for all Staphylococcus spp. and methicillin-resistant Staphylococcus aureus (MRSA), the highest signals were in the definite VRI group. Although there were no definite VRIs with Enterococcus spp. or Klebsiella spp., low signal relative to the quantitative positive control signal was observed in the group without a VRI for 2 of 3 Enterococcus spp.-positive samples and 1 of 2 Klebsiella spp.-positive samples, suggesting possible device colonization.

We calculated the sensitivity and specificity of the assay by using the definite VRI subjects as the case definition and considered only pathogens detectable by the current MassTag PCR assay (i.e., 6 of 6 had true-positive results) and the no VRI subjects as the controls (i.e., 13 of 19 had true-negative results). Using these criteria, the sensitivity of the assay was $100 \%$ (95\% CI 54\%-100\%) and specificity was $68 \%$ (95\% CI $44 \%-87 \%)$. The negative predictive value was $100 \%(95 \%$ CI $75 \%-100 \%)$ and the positive predictive value was 50\% (95\% CI 21\%-79\%).

Antibiotic exposure within 24 hours before CSF sampling, either as part of prolonged prophylaxis for the external ventricular drain placement or as treatment for another site of infection, occurred only in 3 of $9(33 \%)$ of the definite VRI group compared with 15 of 17 (88\%) of the possible VRI group and 14 of $19(77 \%)$ of the no VRI group ( $\mathrm{p}<0.05$, both comparisons). Among the subjects with positive PCR results, 1 of $6(17 \%)$ received antibiotics that were active against at least one of the organisms detected by PCR (and culture) in the definite VRI group compared with 3 of $5(60 \%)$ in the possible VRI group and 2 of $6(33 \%)$ in the no VRI group ( $p>0.5$, both comparisons)(Table 2).

Of the 3 subjects who had more than 1 specimen available, 2 were from the possible VRI group and showed consistent positive PCR results for $S$. aureus. The first patient had MSSA detected in CSF collected 4 days apart, while the second patient had MRSA detected in CSF collected 5 days apart. The agent in both cases was not detected using traditional culture methods. The third subject was from the definite VRI group and had Candida albicans detected in CSF collected 4 days apart by culture and PCR despite directed antifungal therapy.

\section{Discussion}

Several multiplex PCR assays have been designed to obtain an earlier diagnosis than conventional culture methods in cases of acute viral and bacterial meningitis., ${ }^{4,9,13}$ Fewer studies have been published on multiplex approaches for the early diagnosis of VRIs. Banks et al., ${ }^{1}$ developed a strategy that used a broad-range PCR followed by a nested PCR using primers for selected gram-positive bacteria (Propionibacterium acnes, S. aureus, and MRSA) in patients with a clinical suspicion of VRI. Deutch et al. ${ }^{3}$ used a similar approach in patients with and without VRI with a broad-range real-time PCR assay followed by sequencing of the amplicon to identify the pathogen, including grampositive and gram-negative bacteria. The MassTag assay reported here differs from these previous studies by limiting the inherent contamination risk of multistep PCRs through restricting the approach to just 1 round of PCR, and by parallel testing multiple agents through inclusion of specific primers for 11 different bacterial and fungal pathogens and an S. aureus antibiotic resistance gene.

The assay performed well with definitive VRI cases in which a primer for the pathogen was present. One of the strengths of the MassTag PCR platform is that it is relatively straightforward to add additional targets as desired, since only 2 short specific primer sequences are required.

Our assay also detected microbial nucleic acids in $32 \%$ 


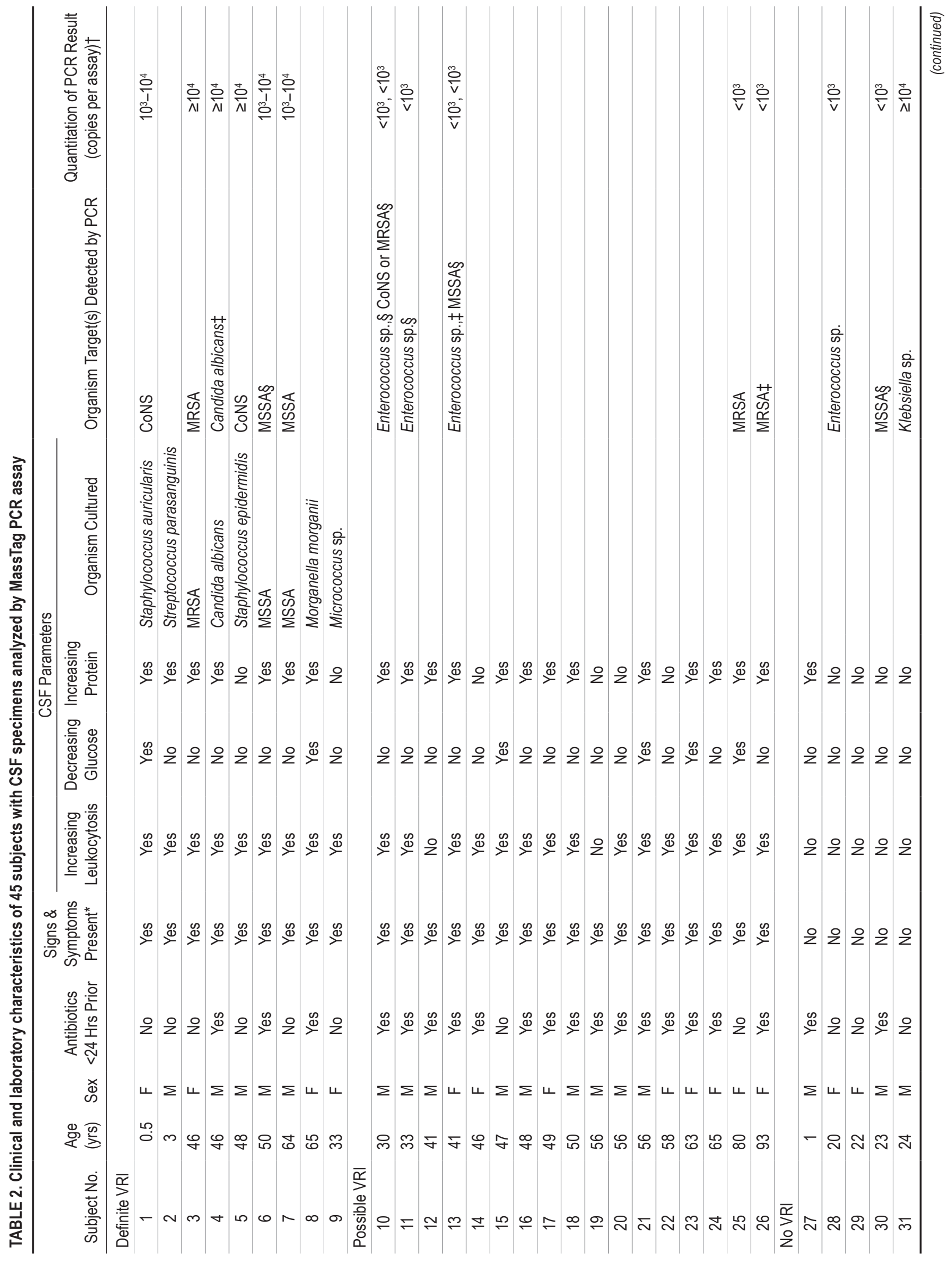




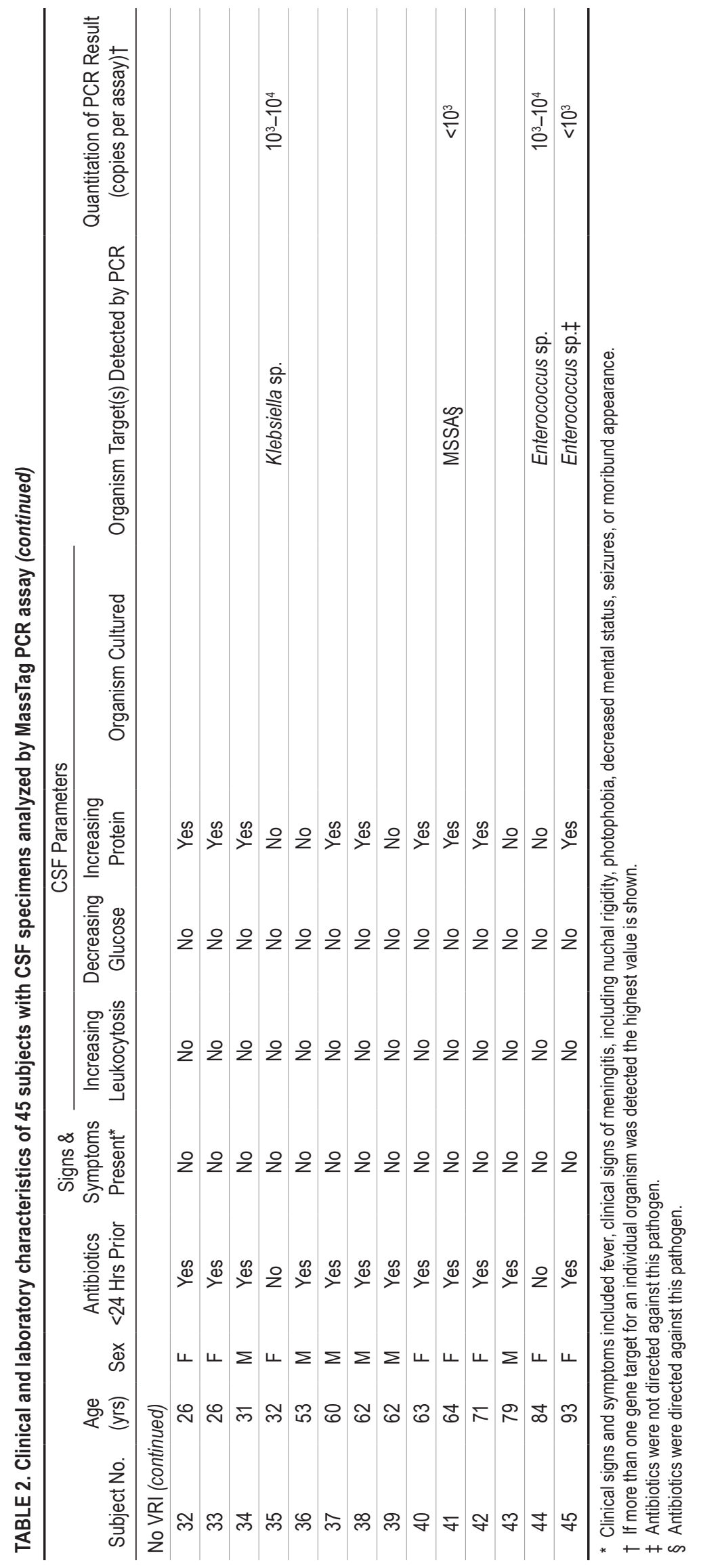


TABLE 3. Evaluation of VRI MassTag panel

\begin{tabular}{|c|c|c|c|}
\hline Infection Category & $\begin{array}{l}\text { No. of Samples } \\
\text { Tested }\end{array}$ & $\begin{array}{l}\text { Positive MassTag } \\
\text { Result }\end{array}$ & Organisms Detected (No.) \\
\hline Definite VRI & 9 & $6(67 \%)^{*}$ & CoNS (2),† MSSA (2), MRSA (1), C. albicans (1) \\
\hline Possible VRI & 17 & $5(29 \%)$ & $\begin{array}{l}\text { MRSA (2), Enterococcus sp. (1), Enterococcus sp. \& MSSA (1), Enterococcus sp. \& MRSA or } \\
\text { CoNS (1) } \ddagger\end{array}$ \\
\hline No VRI & 19 & $6(32 \%)$ & MSSA (2), Enterococcus spp. (3), Klebsiella [KLEB] (2) \\
\hline
\end{tabular}

of subjects without active VRI. Positive PCR results from CSF sampled from ventricular drains in the absence of VRI may indicate device colonization, potential contamination events, or the presence of nonviable bacteria as a consequence of antibiotic treatment. While colonization or contamination was described as a problem encountered in other multiplex PCR VRI approaches, the rate was not reported. ${ }^{3}$ To further differentiate between colonization or contamination, we tested 123 CSF samples from the NYP Clinical Microbiology Service that were collected from subjects without a ventricular drain and without clinical or laboratory evidence of central nervous system infection using standard clinical definitions. ${ }^{11,12}$ Twelve samples (9.8\%) yielded positive signals for bacteria: coagulase-negative Staphylococcus spp. ([CoNS] n = 4, 33\%), CoNS or MRSA $(\mathrm{n}=1,8 \%), \operatorname{MSSA}(\mathrm{n}=2,17 \%)$, Enterococcus spp. $(\mathrm{n}=3,25 \%)$, Klebsiella $\mathrm{sp} .(\mathrm{n}=1,8 \%)$, or Enterococcus $\mathrm{sp}$. and CoNS $(n=1,8 \%)$. In nearly all cases detection was at a low signal level close to the assay cutoff. These data suggest that approximately $10 \%$ of CSF specimens obtained from a clinical microbiology laboratory may potentially produce false-positive results due to contamination during sample collection, microbiology laboratory handling, and/ or PCR processing.

Therefore, in our subjects with a ventricular drain, low positive signals may be due to contamination events, in addition to the possibility of ventricular drain colonization. Another possible explanation for PCR results with a low positive signal and a negative culture is the presence of nonviable bacteria due to antibiotics, from either prolonged cefazolin or other antibiotic therapy, or rifampinand minocycline-coated drains. More precise quantitation of nucleic acid load may be helpful to distinguish between these scenarios; however, this remains to be shown. More recently, Rath et al., described the use of a multiplex PCR, including intrathecal biomarkers (interleukin-6 and lactate). ${ }^{8}$ This approach represents another potential method to distinguish true infection from other causes for the presence of microbial nucleic acid at low levels.

Our study has several limitations. It was performed at a single center, the number of patient samples is relatively small, and the utilization of prolonged antibiotic prophylaxis and/or antibiotic-impregnated catheters was not consistent throughout the study period. Also, CSF samples were frozen prior to nucleic acid extraction, which may have affected genomic DNA integrity; however, samples were stored at $-70^{\circ} \mathrm{C}$ and were not thawed prior to nucleic acid extraction. A potential for sample contamination may be reduced by taking a dedicated CSF sample at the patient's bedside and running the assay in real time with minimal sample handling; however, our results reflect true specimen processing in a clinical setting and thus may be more generalizable. Another limitation was that the current assay did not differentiate between MRSA and CoNS if the mecA gene was the only Staphylococcus sp. target detected, because both MRSA and also some strains of CoNS possess the mecA gene. This can be addressed by adding specific primers to distinguish CoNS in an optimized version of the assay. Additional testing of the assay with a broader range of clinical samples is required to evaluate detection of gram-negative and fungal pathogens. Lastly, our study and other studies of novel diagnostic methods for VRIs, are hampered by the lack of a goldstandard definition for true VRI, since the combination of symptoms, CSF profile, and microbial Gram stain or culture appear insufficient.

Currently, there are no PCR platforms in general use for the microbial diagnosis of VRI. The value of our current assay is also limited given the high sensitivity of PCR and the difficulty of differentiating between true infection and colonization/contamination. Nevertheless, an optimized PCR-based assay may provide timely and economic identification of multiple possible VRI pathogens to help guide choice of antibiotics and duration of therapy, complementing the classic culture approaches.

\section{Conclusions}

The VRI MassTag PCR primer panel performed well in definite VRI, if culture served as the gold standard. In addition, pathogens were identified in $29 \%$ of possible VRI cases. Pathogen nucleic acid was also detected with low signal strength in one-third of cases without a VRI. This possibly reflects ventricular drain colonization, sample contamination, or nonviable organisms due to antibiotic treatment. The lack of clear differentiation between these possibilities renders the current assay of limited clinical value. Nevertheless, in conjunction with clinical and CSF parameters, our multiplex PCR assay can provide timely, economic, and specific identification of multiple common VRI pathogens, improving on current culture-based diagnostics. Further development of the PCR approach, potentially paired with intrathecal biomarkers, can be envisioned to improve differentiation between infection, device colonization, or contamination, and to monitor the efficacy of antibiotic treatment. 


\section{References}

1. Banks JT, Bharara S, Tubbs RS, Wolff CL, Gillespie GY, Markert JM, et al: Polymerase chain reaction for the rapid detection of cerebrospinal fluid shunt or ventriculostomy infections. Neurosurgery 57:1237-1243, 2005

2. Briese T, Palacios G, Kokoris M, Jabado O, Liu Z, Renwick $\mathrm{N}$, et al: Diagnostic system for rapid and sensitive differential detection of pathogens. Emerg Infect Dis 11:310-313, 2005

3. Deutch S, Dahlberg D, Hedegaard J, Schmidt MB, Møller JK, Ostergaard L: Diagnosis of ventricular drainage-related bacterial meningitis by broad-range real-time polymerase chain reaction. Neurosurgery 61:306-312, 2007

4. Favaro M, Savini V, Favalli C, Fontana C: A multi-target real-time PCR assay for rapid identification of meningitisassociated microorganisms. Mol Biotechnol 53:74-79, 2013

5. Forgacs P, Geyer CA, Freidberg SR: Characterization of chemical meningitis after neurological surgery. Clin Infect Dis 32:179-185, 2001

6. Lozier AP, Sciacca RR, Romagnoli MF, Connolly ES Jr: Ventriculostomy-related infections: a critical review of the literature. Neurosurgery 62 (Suppl 2):688-700, 2008

7. Poon WS, Ng S, Wai S: CSF antibiotic prophylaxis for neurosurgical patients with ventriculostomy: a randomised study. Acta Neurochir Suppl 71:146-148, 1998

8. Rath PM, Schoch B, Adamzik M, Steinmann E, Buer J, Steinmann J: Value of multiplex PCR using cerebrospinal fluid for the diagnosis of ventriculostomy-related meningitis in neurosurgery patients. Infection 42:621-627, 2014

9. Shin SY, Kwon KC, Park JW, Kim JM, Shin SY, Koo SH: Evaluation of the Seeplex ${ }^{\circledR}$ Meningitis ACE Detection kit for the detection of 12 common bacterial and viral pathogens of acute meningitis. Ann Lab Med 32:44-49, 2012

10. Stevens EA, Palavecino E, Sherertz RJ, Shihabi Z, Couture DE: Effects of antibiotic-impregnated external ventricular drains on bacterial culture results: an in vitro analysis. J Neurosurg 113:86-92, 2010

11. Tunkel AR, Glaser CA, Bloch KC, Sejvar JJ, Marra CM, Roos KL, et al: The management of encephalitis: clinical practice guidelines by the Infectious Diseases Society of America. Clin Infect Dis 47:303-327, 2008

12. Tunkel AR, Hartman BJ, Kaplan SL, Kaufman BA, Roos KL, Scheld WM, et al: Practice guidelines for the management of bacterial meningitis. Clin Infect Dis 39:1267-1284, 2004

13. Wang X, Theodore MJ, Mair R, Trujillo-Lopez E, du Plessis $\mathrm{M}$, Wolter N, et al: Clinical validation of multiplex real-time PCR assays for detection of bacterial meningitis pathogens. $\mathbf{J}$ Clin Microbiol 50:702-708, 2012

14. Wong GK, Poon WS, Lyon D, Wai S: Cefepime vs. Ampicillin/Sulbactam and Aztreonam as antibiotic prophylaxis in neurosurgical patients with external ventricular drain: result of a prospective randomized controlled clinical trial. J Clin Pharm Ther 31:231-235, 2006

15. Wright K, Young P, Brickman C, Sam T, Badjatia N, Pereira $\mathrm{M}$, et al: Rates and determinants of ventriculostomy-related infections during a hospital transition to use of antibioticcoated external ventricular drains. Neurosurg Focus 34(5):E12, 2013

\section{Author Contributions}

Conception and design: Yin, Tokarz, Briese. Acquisition of data: Yin, Gordon, Tokarz, Whittier, Shah. Analysis and interpretation of data: Yin, Gordon, Tokarz, Briese. Drafting the article: Yin, Gordon. Critically revising the article: Yin, Gordon, Tokarz, Briese, Lipkin, Whittier, Shah, Connolly. Reviewed submitted version of manuscript: Yin, Gordon, Tokarz, Briese, Lipkin, Whittier, Shah. Approved the final version of the manuscript on behalf of all authors: Yin. Statistical analysis: Yin. Administrative/technical/material support: Lipkin, Jain. Study supervision: Yin, Briese.

\section{Correspondence}

Michael Yin, Department of Medicine, Division of Infectious Diseases, 630 W. 168th St., P\&S Box 82, New York, NY 10032. email: mty4@cumc.columbia.edu. 